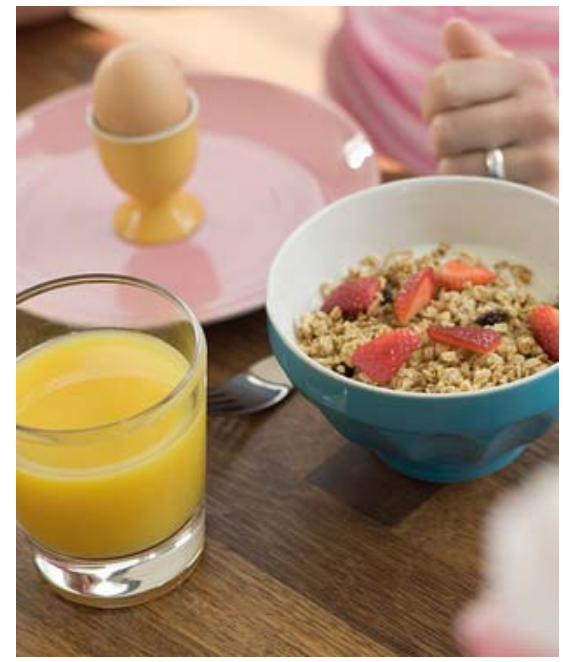

Die Ergebnisse von Uzhova et al. untermauern die Wichtigkeit des Frühstücks und dehnen den Bedeutungsbereich auf die Verringerung der Atherosklerose-Prävalenz aus (Symbolbild; Quelle: Westend61).

\section{Frühstücken senkt kardiovaskuläres Risiko}

Uzhova I et al. The importance of breakfast in atherosclerosis disease: insights from the PESA Study. J Am Coll Cardiol 2017; 70: 1833-42

Das Frühstück hat als erste Mahlzeit des Tages Auswirkungen auf das Sättigungsgefühl, die Appetitregulierung und die tägliche Gesamtenergieaufnahme. Der Verzicht auf ein Frühstück ist mit ungünstigen Auswirkungen auf den Lipidstoffwechsel, Adipositas und Diabetes mellitus assoziiert. Jetzt konnte gezeigt werden, dass auch das
Atheroskleroserisiko bei Personen, die nicht frühstücken, erhöht ist.

Die PESA-Studie (PESA: Progression of Early Subclinical Atherosclerosis) ist eine fortlaufende Beobachtungsstudie, in der Angestellte der Bank Santander in Madrid im Hinblick auf subklinische atherosklerotische Veränderungen untersucht werden. Es wurden Männer und Frauen zwischen 40 und 54 Jahren eingeschlossen, die zu Studienbeginn nicht unter einer kardiovaskulären Erkrankung oder einer chronischen Nierenschädigung litten. Auch Patienten mit Malignom bzw. einer lebenslimitierenden Krankheit und Patienten mit Organtransplantation in der Vorgeschichte wurden nicht in die Studie aufgenommen.

In die aktuelle Querschnittsanalyse gingen die Daten von 4082 Personen ein. Mithilfe eines Fragebogens mussten die Studienteilnehmer angeben, welche Nahrungsmittel sie in welcher Menge und zu welchem Zeitpunkt im Tagesablauf in den letzten 15 Tagen verzehrt hatten. Insgesamt 861 einzelne Ernährungsbestandteile (darunter 184 typisch spanische) standen zur Auswahl. Die Autoren berechneten den prozentualen Anteil des Frühstücks an der Gesamtenergieaufnahme und klassifizierten die Studienpopulation in folgende Gruppen: 
- Frühstück mit hohem Energiegehalt (>20\% der täglichen Gesamtenergieaufnahme)

- Frühstück mit niedrigem Energiegehalt (5-20\% der täglichen Gesamtenergieaufnahme)

- kein Frühstück (morgendliche Energieaufnahme < $5 \%$ der täglichen Gesamtenergieaufnahme)

Als Screeninguntersuchungen wurde den Studienteilnehmerinnen und -teilnehmern eine dopplersonografische Untersuchung der Karotiden, der infrarenalen Aorta sowie der Becken- und Beinarterien und eine Bestimmung des koronaren KalziumScores mittels Computertomografie angeboten.

Nur 3\% der Studienteilnehmer verzichteten auf ein Frühstück, 69\% nahmen ein Frühstück mit niedrigem und $28 \%$ ein Frühstück mit hohem Energiegehalt zu sich. Personen, die das Frühstück ausließen, konsumierten durchschnittlich höhere Gesamtenergiemengen im Tagesverlauf, nahmen mehr tierische Proteine und höhere Mengen an Cholesterin zu sich, hatten einen geringen Ballaststoffanteil in ihrer Ernährung, tranken mehr alkoholische und mehr gesüßte Getränke und rauchten häufiger. Außerdem wiesen Nichtfrühstücker häufiger kardiovaskuläre Risikofaktoren auf, wie Adipositas, Hypercholesterinämie und arterielle Hypertonie.

Bei insgesamt 62,5\% der Studienteilnehmer zeigte sich eine subklinische Atherosklerose (Plaques in mindestens einer der beiden Karotiden bzw. Becken-/Beinarterien oder der Aorta bzw. koronarer Kalzium-Score $>0$ ) und bei 60,3\% eine Atherosklerose außerhalb der Koronararterien. Eine generalisierte Atherosklerose (mindestens 4 der 6 Lokalisationen betroffen) konnte bei 13,4\% nachgewiesen werden. Studienteilnehmer, die das Frühstück ausließen, hatten auch nach Adjustierung an traditionelle Risikofaktoren im Vergleich zu Personen mit hochkalorischem Frühstück eine erhöhte Atherosklerose-Prävalenz in Gefäßgebieten außerhalb der Herzkranzgefäße $(O R$ 1,55) und eine erhöhte Prävalenz für eine generalisierte Atherosklerose (OR 2,57).
FAZIT

Die Autoren vermuten, dass der nachgewiesene negative Effekt eines fehlenden Frühstücks zum Teil über einen generell ungesunden Lebensstil zu erklären ist. Ein reichhaltiges Frühstück verringere aber auch den Nüchternblutzucker sowie den Insulin- und den Ghrelin-Serumspiegel und schütze so möglicherweise vor einer Überalimentation. Die Daten untermauern die Bedeutung einer gesunden Ernährungsweise mit einem energiehaltigen Frühstück, so die Autoren.

Dr. Katharina Franke, Darmstadt 